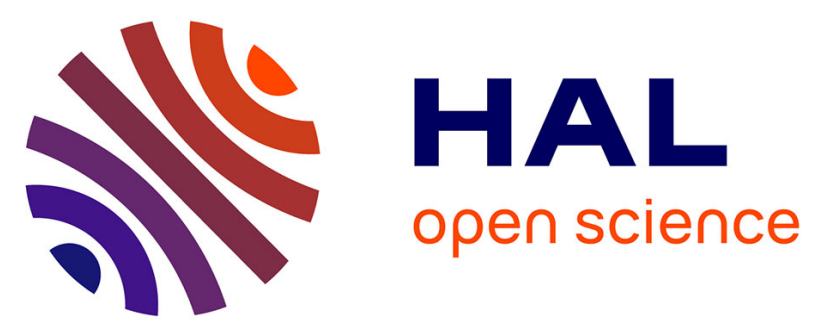

\title{
Mechanical Exfoliation of Select MAX Phases and Mo 4 Ce 4 Al 7 C 3 Single Crystals to Produce MAXenes
} Athanasios Gkountaras, Youngsoo Kim, Johann Coraux, Vincent Bouchiat, Simone Lisi, Michel W Barsoum, Thierry Ouisse

\section{To cite this version:}

Athanasios Gkountaras, Youngsoo Kim, Johann Coraux, Vincent Bouchiat, Simone Lisi, et al.. Mechanical Exfoliation of Select MAX Phases and Mo 4 Ce 4 Al 7 C 3 Single Crystals to Produce MAXenes. Small, 2020, 16 (4), pp.1905784. 10.1002/smll.201905784 . hal-02442870

\section{HAL Id: hal-02442870 \\ https://hal.science/hal-02442870}

Submitted on 6 Nov 2020

HAL is a multi-disciplinary open access archive for the deposit and dissemination of scientific research documents, whether they are published or not. The documents may come from teaching and research institutions in France or abroad, or from public or private research centers.
L'archive ouverte pluridisciplinaire HAL, est destinée au dépôt et à la diffusion de documents scientifiques de niveau recherche, publiés ou non, émanant des établissements d'enseignement et de recherche français ou étrangers, des laboratoires publics ou privés. 


\section{Mechanical Exfoliation of select MAX phases and $\mathrm{Mo}_{4} \mathrm{Ce}_{4} \mathrm{Al}_{7} \mathrm{C}_{3}$ single crystals to produce MAXenes}

Athanasios Gkountaras, Youngsoo Kim, Johann Coraux, Vincent Bouchiat, Simone Lisi, Michel W. Barsoum*, Thierry Ouisse*

\section{A. Gountaras}

Univ. Grenoble-Alpes, CNRS, Inst. NEEL \& LMGP, F-38042 Grenoble, France

Y. Kim

Univ. Grenoble Alpes, CNRS, Grenoble INP, LMGP, F-38000 Grenoble, France

J. Coraux, V. Bouchiat, S. Lisi

Univ. Grenoble-Alpes, CNRS, Inst. NEEL, F-38042 Grenoble, France

M. W. Barsoum

Department of Materials Science and Engineering, Drexel University, Philadelphia, PA 19104, USA

T. Ouisse

Univ. Grenoble Alpes, CNRS, Grenoble INP, LMGP, F-38000 Grenoble, France

Email: Thierry.ouisse@grenoble-inp.fr barsoum@drexel.edu

Keywords: MAX phases; Two-dimensional systems; Exfoliation; Electric Force Microscopy

Abstract: MXenes - two dimensional carbides/nitrides derived from their bulk nanolamellar $M_{n+1} A X_{n}$ phase counterparts - are, for the most part, obtained by chemical etching. Despite the fact that the M-A bonds in the MAX phases are not weak, in this work we demonstrate that relatively large MAX single crystals can be mechanically exfoliated using the adhesive tape method to produce flakes whose thickness can be reduced down to half a unit cell. The exfoliated flakes, transferred onto $\mathrm{SiO}_{2} / \mathrm{Si}$ substrates, are analyzed using electric force microscopy, EFM. No appreciable variation in EFM signal with flake thickness is found. EFM contrast between the flakes and $\mathrm{SiO}_{2}$ not only depends on the contact surface potential, but also on the local capacitance. The contribution of the latter can be used to show the metallic character - confirmed by 4-contact resistivity measurements - of even the thinnest of flakes. Because the A-layers are preserved, strictly speaking we are not dealing with MXenes, but rather MAXenes. This is important in the case where the " $\mathrm{A}$ " layers contain magnetic elements such as $\mathrm{Mo}_{4} \mathrm{Ce}_{4} \mathrm{Al}_{7} \mathrm{C}_{3}$ - whose structure is a derivative of the MAX structure. 
Discovered in 2011, ${ }^{[1]}$ MXenes are 2D carbides and nitrides, which are typically synthesized from the machinable, layered, ternary phases known as the MAX phases. ${ }^{[2],[3]}$ The stoichiometry of a typical MAX phase is $M_{n+1} A X_{n}$, where $M$ stands for an early transition metal, $A$ belongs mostly to groups 13 to 15 and $X$ $=\mathrm{C}$ and/or $\mathrm{N}$ and $\mathrm{n}=1,2$ or $3 .{ }^{[2]}$ MXenes - typically produced by etching the Al layers of Al-based MAX phase powders ${ }^{[[1],[4]]}$ stand out by their high metallicity, ${ }^{[5],[6]}$ hydrophilicity and dispersibility in polar solvents. ${ }^{[7],[8]}$ They present unique and exciting properties and characteristics of high technological potential for applications such as supercapacitors, ${ }^{[9],[10]}$ electromagnetic shielding, ${ }^{[11]}$ bio-sensing, ${ }^{[12]}$ electrode materials for Li and multivalent ion batteries, ${ }^{[13],[14],[15]}$ electrochemical catalytic surfaces, ${ }^{[15],[16]}$ hydrogen storage, ${ }^{[17]}$ thermoelectric materials, ${ }^{[18]}$ sensors ${ }^{[12]}$ and photo-thermal medical devices for tumor eradication, ${ }^{[19]}$ among many others.

In sharp contradistinction to graphite, and other weakly bonded solids, the main reason MXenes have to date been produced by chemical exfoliation, ${ }^{[1]]]}$ is that the bonds between the $M$ and $A$ layers are far from weak. ${ }^{[2]}$ To mechanically exfoliate the MAX phases requires the breaking of strong, ductile, metallic bonds. The lack of, until recently, large enough MAX single crystals, SCs, was also an impediment to attempting mechanical exfoliation. However, given that at least some MAX phase compositions are now routinely grown from high temperature solutions in SC form, ${ }^{[20],[21],[22]}$ has removed this impediment and prompted this work.

The aim of this work, is to report on how the adhesive tape method, ${ }^{[23]}$ - applied to MAX SCs of an appropriate size and thickness - can be used to mechanically exfoliate the MAX phases down to thicknesses of less than a unit cell of the starting crystal structure. These flakes were then successfully transferred onto

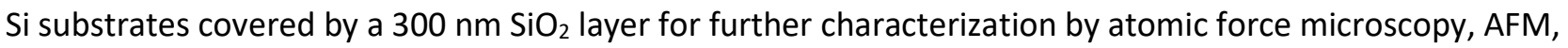
electrostatic force microscopy, EFM and Kelvin Probe Force Microscopy, KPFM. As shown herein, we successfully mechanically exfoliated $\mathrm{V}_{2} \mathrm{AIC}, \mathrm{Ti}_{2} \mathrm{SnC}, \mathrm{Cr}_{2} \mathrm{AlC}$ and $\mathrm{Mo}_{4} \mathrm{Ce}_{4} \mathrm{Al}_{7} \mathrm{C}_{3}$. The quaternary, $\mathrm{Mo}_{4} \mathrm{Ce}_{4} \mathrm{Al}_{7} \mathrm{C}_{3}$, belongs to a new class of nanolaminated phases that are $\mathrm{MAX}$ phase derivatives in that $\mathrm{Mo}_{4} \mathrm{Ce}_{2} \mathrm{C}_{3}$ layers are separated from each other by $\mathrm{Al}_{7} \mathrm{Ce}_{2}$ layers. ${ }^{[24]}$ These phases are quite interesting in that they are ferromagnetic at $\mathrm{T}<10 \mathrm{~K} \cdot{ }^{[24]}$ In $\mathrm{Mo}_{4} \mathrm{Ce}_{4} \mathrm{Al}_{7} \mathrm{C}_{3}$, magnetic ordering has been shown to arise from a fraction of 
the $\mathrm{Ce}$ atoms that reside in the $\mathrm{Al}_{7} \mathrm{Ce}_{2}$ layers that interleaf the $\mathrm{Mo}_{4} \mathrm{Ce}_{2} \mathrm{C}_{3} \mathrm{M}_{2} \mathrm{X}$-like layers. ${ }^{[24]}$ This is important in the context of this work because the produced flakes, for the most part, retain either their Al-layers in case of the MAX phases or the $\mathrm{Al}_{7} \mathrm{Ce}_{2}$ layers in the $\mathrm{Mo}_{4} \mathrm{Ce}_{4} \mathrm{Al}_{7} \mathrm{C}_{3}$ case. Strictly speaking, our ultrathin films are therefore not MXenes, but MAXenes.

Before describing our results, it is important to note that to reach the point described in this work, required significant time and effort. The selected process is described in Section S1. Similar to graphene, when MXene flakes are transferred on a $300 \mathrm{~nm}$ silica layer on $\mathrm{Si}$, they can be directly observed under an optical microscope, OM. ${ }^{[25]}$ As shown herein, the flake area varied from a very few $\mu \mathrm{m}^{2}$ to some tens of $\mu \mathrm{m}^{2}$.

Figure 1 presents AFM images of thin flakes - obtained by mechanical exfoliation using the adhesive tape technique - of $\mathrm{V}_{2} \mathrm{AlC}, \mathrm{Ti}_{2} \mathrm{SnC}, \mathrm{Cr}_{2} \mathrm{AlC}$ and $\mathrm{Mo}_{4} \mathrm{Ce}_{4} \mathrm{Al}_{7} \mathrm{C}_{3}$. The presence of glue residue at the bottom and top of the flakes limited the accuracy of our estimation of the flake thicknesses. Nevertheless, the measured values, more often than not, were quite close to integer values of half unit cells. Hereafter, the measured layer thicknesses will be given in multiples of $L$, where $L$ is the thickness of a unit cell. Measuring flake height shows that the thickness can be reduced down to a unit cell, or $1 \mathrm{~L}$, and in some cases even down to $0.5 \mathrm{~L}$ (see Figure $1 b$ ). A MAX unit cell contains two $M_{n+1} C_{n}$ layers separated by an A plane. Flakes with the largest areas (>20 $\mu \mathrm{m}$ in lateral size) were in general thicker (from $5 \mathrm{~L}$ to $10 \mathrm{~L}$ ) than smaller ones. When the lateral size was closer to a few $\mu \mathrm{m}^{2}$, the flake thicknesses were closer to $1 \mathrm{~L}$ and $2 \mathrm{~L}$. In contrast with chemically exfoliated flakes, that are terminated by $\mathrm{O}-,-\mathrm{OH},-\mathrm{F}$ or other anions, in our flakes the $\mathrm{Al}$ atoms remain, together with the presence of glue residue (see Figure 1). Removing this glue residue was a major challenge in this work (see Section S1). Before addressing our attempts to remove it, we wanted to understand how mechanical cleavage affected the MAX structure. To that effect we cleaved millimeter-sized $\mathrm{Cr}_{2} \mathrm{AIC} \mathrm{SCs}$ under ultrahigh vacuum ( $\left.10^{-10} \mathrm{mbar}\right)$, and analyzed the cleaved surface in situ, without exposure to air, with scanning tunneling microscopy, STM. Even under such clean conditions, stable imaging conditions were difficult to achieve, presumably due to the reactivity of the cleaved surface with, e.g., the scanning tip of the microscope. Typical STM images - as far as we are aware, the first of their kind - of the surface are 
shown in Figures $1 \mathrm{~g}$, $\mathrm{h}$ and $\mathrm{i}$. The atomic resolution images indicate that the hexagonal, closed-packed (hcp) lattice of the $\mathrm{Cr}$ atoms is preserved (Figure $1 \mathrm{~g}$ ).

STM images at a larger scale (Figures $1 \mathrm{~h}$ and $1 \mathrm{i}$ ) clearly show that the surface is separated into two nested or interpenetrating domains, with different apparent heights. It is thus tempting to attribute this mottled structure to uneven distribution of the Al atoms of the cleaved atomic planes. Since it is reasonable to assume that the $\mathrm{Al}$ atoms are distributed roughly equally on each face of the fractured surfaces, surface reconstruction, or Al clustering, could then lead to the formation of the two distinct regions observed. Here we note that the $\mathrm{Al}$ atoms are in fact more strongly bonded to the $\mathrm{M}$ atoms than their in-plane neighbors, ${ }^{[3]}$ which in principle favors their equal repartition between both fractured faces. When exfoliation is conducted in air, we expect these atoms to very rapidly react with oxygen, $\mathrm{O}$, to form $\mathrm{Al}-\mathrm{O}$ clusters.

In the MAX phases, the largest contribution to the density of states (DOS) at the Fermi level $\left(E_{F}\right)$ is that of the d-orbitals of the $\mathrm{M}-\mathrm{Cr}$ in this case - atoms. ${ }^{[[2],[26]]}$ It follows that the Al-free surfaces should appear closer to the tip than the Al-covered regions. Said otherwise, the tunneling current setpoint (constant-current imaging is used here) value is obtained at a larger distance when the DOS is higher. We therefore tentatively attribute the existence of the darker domains to disordered Al or Al-O clusters and the brighter ones to the Cr-rich surfaces. In principle, one should thus be able to distinguish a regular hcp atomic lattice in the latter areas, as observed and depicted by a hexagon on lower left side of Figure $1 \mathrm{~g}$. These observations notwithstanding, much more STM work, some of which is ongoing is needed to flesh out some of our conjectures.

EFM and KPFM are routinely used for assessing electrical surface properties (see, e.g., Refs. ${ }^{[27],[28],[29]}$ for EFM and ${ }^{[30],[31],[32]}$ for KPFM). They allow for the detection of charges or surface potentials with high sensitivity ${ }^{[28],[33],[34]}$, and do not require any additional processing for assessing flake properties once they are transferred onto a Si substrate with a top $\mathrm{SiO}_{2}$ layer. The $\mathrm{Si}$ substrate acts as a back conducting plane. Both methods have been widely used in the case of $2 \mathrm{D}$ materials. ${ }^{[35],[36],[37],[38],[39],[40],,[41],[42],[43],[44],,[45]}$ In the EFM lift mode and maintaining a constant probe distance, $z$, the gradient of the electric force between the tip and the sample is simply added to the cantilever's effective spring constant $k$. This 
accordingly modifies the resonance frequency and leads to the well-known phase dependence of the mechanically excited oscillation of the cantilever, given by ${ }^{[35]}$

$$
\Delta \Phi=-\frac{Q}{2 k} \frac{\partial^{2} C}{\partial z^{2}}\left(V_{0}-\phi_{t i p}+\phi_{B}+\Delta \phi_{S}\right)^{2}
$$

In the presence of our conducting flakes (Figure 2a), C reduces to the tip-flake capacitance $C_{M}$ (as demonstrated in Section S2). In the absence of any flakes, viz. in the presence of only the $\mathrm{SiO}_{2}$ layer (Figure 2b), $\mathrm{C}$ is the tip-Si substrate capacitance $C_{\mathrm{SiO} 2} . \phi_{\mathrm{tip}}, \phi_{\mathrm{B}}$ and $\Delta \phi_{\mathrm{S}}=\phi_{\mathrm{S} 1}-\phi_{\mathrm{S} 2}$ are the tip surface potential, the Si surface potential at the $\mathrm{Si}_{-} \mathrm{SiO}_{2}$ interface and the difference of surface potentials between the top and bottom of a given flake, respectively. $V_{0}$ is the externally applied voltage. The influence of adsorbed surface charges (e.g., charges due to triboelectrification of the glue residues at the peeling point, ${ }^{[43]}$ charges in the $\mathrm{SiO}_{2}$ layer, ${ }^{[34]}$ adsorbed water molecules, ${ }^{[40],[46],[47]}$ etc.) can be formally incorporated under the form of additional shifts of those surface potential values. Equation 1 displays the well-known parabolic dependence of the phase shift on $V_{0}$, at a minimum when $V_{0}$ is equal to the surface contact potential difference, $\mathrm{V}_{\mathrm{CPD}}{ }^{[32]}$ Henceforth the configurations shown in Figs. $2 \mathrm{a}$ and $\mathrm{b}$ will be referred to as Metal-AirMetal (MAM) and Metal-Air-Dielectric-Metal (MADM) configurations, respectively.

For an ideal structure, a surface potential-induced contrast between the flake and the bare $\mathrm{SiO}_{2}$ surface is to be expected only when there is a surface potential difference between the top and the bottom of the flakes, viz. $\Delta \phi_{s} \neq 0$. In the case of graphene, the low DOS renders thin flakes non-ideal conductors, so that very few-layered flakes differ from their thicker counterparts. This is not observed here. When positioning the tip above a bare $\mathrm{SiO}_{2}$ surface, two changes are expected: first, a contrast in surface potential between $\mathrm{SiO}_{2}$ and the flake. This is in general the main contribution to phase contrast. However, in our case we are also interested in establishing whether the flakes have metallic character or not. This information can be obtained from the curvature of the parabola. Although this parameter is seldom studied in the literature of flakes, its value is directly connected to the tip-sample capacitance (see Equation 1). Due to the non-planar geometry of the tip, and to the insulating character of both air and $\mathrm{SiO}_{2}$, we do not expect the $\mathrm{SiO}_{2}$ top surface to be fully equipotential, and thus a contrast in parabola curvature is in principle possible. 
Given that the dielectric constant $\varepsilon$ of $\mathrm{SiO}_{2}$ is appreciably larger than that of air, it is important to establish whether a capacitance contrast is possible. A key parameter is $\beta=\left(\varepsilon-\varepsilon_{0}\right) /\left(\varepsilon+\varepsilon_{0}\right) .{ }^{[48],[49]}$ When $\beta=0$, the dielectric layer is replaced by air, and for $\beta=1$ the conventional, tip-metal plane configuration is recovered. ${ }^{[48]}$ This problem has been recently treated in the sphere-plane geometry. ${ }^{[48],[49]}$ This configuration compares well to the AFM configuration at short distances (sphere radius $\mathrm{R}>\mathrm{z}$ and $\mathrm{d}<<\mathrm{R}$ ). ${ }^{[48],[49]}$ Unfortunately, in our case, with a tip apex of radius around $20 \mathrm{~nm}$, and $\mathrm{k}=2.8 \mathrm{~N} / \mathrm{m}$ or k $=40 \mathrm{~N} / \mathrm{m}$, we always have to measure EFM in conditions where $\mathrm{z}$ is, more often than not, larger than R. In that case, the "stray" capacitance generated by the AFM cone rapidly becomes the prevailing contribution to the capacitance and its derivatives (Figure 2d). ${ }^{[50],[51]}$ Currently there is no available model for computing the cone contribution in the case of the MADM configuration (viz. Figure 2a). In spite of its quantitative inaccuracy for $z$ larger than $\mathrm{R}$, we are thus reduced to rely on computing the capacitance using the sphere-plane model proposed by Sadhegi et al. ${ }^{[48]}$ Capacitance calculation is achieved by generating and summing nested series of image charges of decreasing value, until a satisfying convergence is reached. ${ }^{[48]}$ Here we just use our characteristic dimensions as inputs. Results are shown in Figure 2c. Although the capacitance values of the flake and top $\mathrm{SiO}_{2}$ configurations (Figure $2 \mathrm{c}$ ) as well as their first derivative, which gives the force (Figure $2 \mathrm{~d}$ ), differ slightly when $z \gg>R$, they exhibit a noticeable departure not only at short distances, but also in the medium range ( $z>R$ but smaller than cone length). The second derivative gives $\alpha$, the ratio of the parabola curvature between the two configurations. The latter substantially departs from unity (see Figure $3 \mathrm{~d}$ ). Of course we expect the sphere configuration to become very unprecise for medium distances and above (see, e.g., Figure $2 \mathrm{~d}$ in the flake case, for which the dielectric layer plays no role, so that a good analytical approximation of the sphere+cone configuration, developed by Hudlet et al., ${ }^{[50]}$ can be used and compared to the sphere model). Both models approach one another only when $z<R$. Nevertheless, predicting an appreciable contrast between MAM (Fig. 1a) and MADM (Fig. 1b) configurations in the sphere approximation leaves hope for a contrast to be observed even in the sphere + cone configuration. In the following, we show that this contrast can indeed be observed. A first useful experimental verification consists in checking that the measured capacitance values are reasonable. In the MADM configuration, we 
expect the capacitance variation to be that due to a simple tip-metal plane configuration. In this case we can safely use the analytical formula proposed by Hudlet et al. ${ }^{[50]}$ The second derivative of the capacitance is a complicated, but fully analytical, expression of $z$, quality factor $Q$, spring constant $k$, cone angle $\theta$ and apex radius $R .{ }^{[[50]]}$ By assuming $R=20 \mathrm{~nm}, \theta=10^{\circ}, \mathrm{k}=2.8 \mathrm{~N} / \mathrm{m}$ (spring constant value given by the vendor), and the measured quality factor, $Q=205$, into Hudlet et al. formula, ${ }^{[50]}$ we obtained the result shown in Figure2e, where the solid line corresponds to the analytical model. ${ }^{[50]}$ The dashed line is generated by averaging the values computed with the same model over a moving interval equal to the tip oscillation amplitude. As seen from that figure, the agreement with the measured values is excellent, especially when the averaging technique is used. It therefore sounds reasonable to seek some experimental evidence of a curvature contrast, as indeed observed (see below).

Figure $2 \mathrm{f}$ presents an AFM map of a mechanically exfoliated $\mathrm{Cr}_{2} \mathrm{AlC}$ sample. Figure $2 \mathrm{~g}$ shows the corresponding EFM image. Two features are salient: First, a comparison of both images makes it amply clear that there is no apparent dependence of the EFM signal on flake thickness. The same is also true of $\mathrm{V}_{2} \mathrm{AlC}$ flakes, in which case the thickness was as low as $1 \mathrm{~L}$ (Figure $2 \mathrm{~h}, \mathrm{I}, \mathrm{j})$. Here again there is no correlation between AFM maps (Figure 2i) and either KPFM (Figure 2h) or EFM (Figure 2j) maps confirming that the KPFM/EFM signals are quite a weak function of flake thickness. These results already indicate that the flakes remain metallic down to the monolayer level: Had the thinner flakes been converted to an insulating oxide it would have been reasonable to assume their contrast would differ from the thicker conductive flakes, which is not observed. Secondly, the glue residue, clearly recognizable on the topographic image of Figure $2 \mathrm{f}$, also leads to a distinct and local variation of the EFM signal in Figure $2 \mathrm{~g}$ (this sample was deliberately chosen because of its large amount of glue residue). This contrast difference arises because either the glue residue regions are charged, or they modify the surface potential of the flakes.

For all surfaces studied, we systematically measured phase parabolas as a function of $z$ for both MAM and MADM configurations. A typical measurement on a $\mathrm{Cr}_{2} \mathrm{AlC}$ flake at a $\mathrm{z}$ of $75 \mathrm{~nm}$ is shown in Figure $3 a$. Plots of $\Delta \mathrm{V}_{\mathrm{CPD}}$ for various $\mathrm{z}$ values are shown in Figure $3 \mathrm{~b}$, where sample to sample variability is obvious. The scatter arises from a multitude of parameters such as flake annealing conditions used for removing the 
glue residue, $\mathrm{MAX}$ chemistry, $\mathrm{SiO}_{2}$ surface preparation prior to flake transfer, residual glue on top of the flakes, relative humidity (HR) during the EFM experiments, among others. For example, if different amounts of glue are present on the bottom or top of the flakes, then, in principle, a surface potential contrast should be observed. Annealing conditions can also contribute to an asymmetry between top and bottom, because the reactivity of the glue on the flake's bottom is not necessarily the same as on its top. In short no useful information can be gleaned from the results shown in Figure 3b. These comments notwithstanding, making use of the capacitance analysis presented above we can nevertheless show that the flakes are conductive as follows.

Figure $3 \mathrm{c}$ compares parabolas measured, at various $\mathrm{z}$ values, on a $\mathrm{V}_{2} \mathrm{AlC}$ flake and a bare $\mathrm{SiO}_{2}$ surface. For each of the measured parabolas, we extracted the curvature and $V_{C P D}$ by least-square fitting. We quantify the contrast extracted from the parabolas' fits by the ratio $\alpha$ between the parabola curvatures of the two surfaces (Figure 3d), down to the shortest distances attainable without getting in a repulsive interaction regime with the sample surface. As seen in Figure $3 d$, here again there is a strong sample variability. Three responses were typically observed, however. In some cases, $\alpha$ remained close to unity at all distance indicating no contrast differences between MAM and MADM configurations In some cases, $\alpha>$ 1 and increases with decreasing $z$ values, an observation incompatible with the model presented above. In a third set of measurements, a noticeable decrease in $\alpha$ with increasing $z$ was observed (in qualitative agreement with the model). Within these responses, we also observed various gradations (see Figure $3 \mathrm{~d}$ ), which cannot be explained solely by changes in MAX phase composition and/or annealing conditions. A fact common to all samples, however, is that for large $z$ values, $\alpha$ always tended to 1 , meaning that at large tipsample distances no contrast differences between bare and covered surfaces were visible.

We conjectured that the frequent lack of contrast - and "inverted" contrast (i.e. $\alpha>1$ ) sometimes observed in Figure $3 d$ - was due to the presence of adsorbed water molecules on the $\mathrm{SiO}_{2}$ surfaces, if they were hydrophilic and the relative humidity $(\mathrm{RH})$ was high. ${ }^{[46]}$ Note that even a $15 \% \mathrm{RH}$ results in two monolayer-thick adsorbed water layers, ${ }^{[46]}$ and that the hydrophilic/hydrophobic character may substantially vary with time. ${ }^{[47]}$ To test this conjecture, we prepared two $\mathrm{SiO}_{2}$ surfaces: one hydrophilic, the 
other hydrophobic (see section S3). Experimental $\alpha$ values for $\mathrm{V}_{2} \mathrm{AlC}$ flakes reported on both surfaces are shown in Figure 4a. Figure $4 b$ shows some of the parabolas used to obtain the results shown in Figure 4a. The contact potential difference is shown in Figure $4 \mathrm{c}$. A typical plot used to extract $\alpha$ and $\Delta \mathrm{V}_{\mathrm{CPD}}$ is shown in Figure $4 \mathrm{~d}$. All measurements were acquired on the same day where the RH was measured between 45 and $47 \%$, so it is reasonable to assume that the hydrophilic substrates were covered by substantial water adlayers. ${ }^{[46]}$ Figure 4a clearly demonstrates that for the hydrophobic $\mathrm{SiO}_{2}$ surfaces, the expected drop in $\alpha$ at the shortest distances is observed for all measured samples (see also Figure 4b). Here again as expected, all curves converge towards 1 for larger $z$ values. Further, extracting the contrast $\Delta \mathrm{V}_{\mathrm{CPD}}=\mathrm{V}_{\mathrm{CPD}}$ (flake)$\mathrm{V}_{\mathrm{CPD}}\left(\mathrm{SiO}_{2}\right)$ from the parabola fits also shows that values obtained from the hydrophobic substrates are always larger than those of the hydrophilic ones (Figure 4c). This is in line with the experimental observation that the surface potential of $\mathrm{SiO}_{2}$ surface increases with increasing thickness of adsorbed water layers. ${ }^{[46]}$ These are important results because the capacitance contrast between the $\mathrm{SiO}_{2}$ surface and the flakes, with $\alpha<1$ and in which $\alpha$ decreases with decreasing $z$, indicates that even the thinnest of them, are metallic in nature, an important conclusion. EFM measurements can thus probe the conducting nature of the flakes.

Final verification of this idea, was achieved by measuring the resistivity, $\rho$, of selected flakes as a function of flake thickness (Fig. 4f). The $\rho$ value was extracted using a 4-contact configuration on flakes with homogeneous thicknesses, using the average width, lengths and thicknesses defined by the flake and the distance between the inner processed metal lines as inputs (some uncertainty is introduced by the fact that the sample edges are never straight and parallel). Figure 4e shows OM micrographs of two such 4-contact configurations. Figure $4 \mathrm{f}$ is a plot of the apparent $\rho$ as a function of the flake thickness for several phases. With the exception of the $\mathrm{V}_{2} \mathrm{AlC}$ results, for all other compositions an abnormal increase of the apparent $\rho$ with thickness is observed. Why this is not the case for $\mathrm{V}_{2} \mathrm{AlC}$ is not totally clear at this time, but the fact that for this composition the measurements were carried out on thinner flakes on average is a reasonable conjecture. The extracted $\rho$ values can also be increasingly underestimated with increasing flake thicknesses if interlayer defects, most likely delaminations between layers, are present, for the true 
thickness is then also lower than the one assumed which, in turns, leads to an overestimation in $\rho$. This happens if the contacts are ohmic on top but not at the edges, possibly because (i) flakes are more prone to oxide formation at the edges than on top (a recurrent problem with 2D flakes) and/or (ii) depending on flake edge angle, metal deposition is not conformal. In both cases, current is then just injected at the top. Then the possible reasons why apparent resistivity increases with thickness are (i) partial delamination of intermediate layers and/or (ii) high transport anisotropy of some phases, as previously evidenced in bulk materials. ${ }^{[52],[53]}$ Taking these considerations into account, it follows that the lowest $\rho$ values are more representative of the true $\rho$ values. Table 1 summarizes the inplane resistivities of $\mathrm{V}_{2} \mathrm{AIC}$ and $\mathrm{Cr}_{2} \mathrm{AIC}$ measured here and previously. For $\mathrm{V}_{2} \mathrm{AlC}$, the values measured herein are of the same order as polycrystalline samples. They are however, significantly higher than those measured on macroscopic single crystal samples. The exact reason for the discrepancy is unknown at this time. The first possibility is that the values measured herein are still those of defective crystals. The second is that reducing the dimension along the c-axis results in electron scattering. The situation for $\mathrm{Cr}_{2} \mathrm{AlC}$ is comparable for the same reasons. These results show that a proper estimation of the conductivity would require developing a more complex process with lateral contacts, in order to ensure a contribution from all layers. ${ }^{[56]}$ This is left for future work. This last point is important because DFT calculations by Sankaran et al. have suggested that the MAX phases in general, and $\mathrm{V}_{2} \mathrm{AIC}$ in particular, can be good candidate materials for the next generation interconnects, beyond $\mathrm{Cu}$ and even $\mathrm{Ru} .{ }^{[57]}$ Before concluding we note that our MAXenes are different from the very thin metal carbide single crystals grown by CVD. ${ }^{[58]}$ In the latter, the minimal thickness obtained was larger than a unit cell. More importantly, they do not contain the A-layers. In summary

1/ we show, for the first time, that mechanical exfoliation using the adhesive tape method can be used on nanolamellar phases where the bonding is not weak. It thus opens the door for others to try and exfoliate layered solids that, to date, have been deemed impossible to mechanically exfoliate because of the interlayer strengths. 
2/ One can now produce MAXenes, the MXenes counterparts of which have not been possible by chemical etching such as $\mathrm{Cr}_{2} \mathrm{AlC}$.

3/ One can apply the exfoliation process independently of the nature of the A element. For example, to date $\mathrm{Ti}_{2} \mathrm{SnC}$ has been unetchable, but as shown herein can be mechanically exfoliated.

4/ It is now possible to produce few layers-thick MX layers wherein the A layers remain. This is of a considerable interest in the case of more "exotic" MAX phases, such as the $\mathrm{Mo}_{4} \mathrm{Ce}_{4} \mathrm{Al}_{7} \mathrm{C}_{3}$ ferromagnetic phase. $^{[24]}$

5/ Like in graphene, it is reasonable to assume that the mechanically exfoliated flakes would be significantly less defective than those that were obtained by etching and thus will allow for a more in-depth understanding of intrinsic electronic properties of these new materials.

\section{Experimental Section}

Centimeter-sized SCs can be cleaved, but they cannot be mechanically exfoliated (see Section S1), so that crystals $100 \mu \mathrm{m}-300 \mu \mathrm{m}$ in lateral size were selected by sieve shaking (see Section S1) and exfoliated with a natural rubber or synthetic-based adhesive tape with adhesive strength around 4 to $5 \mathrm{~N} / \mathrm{m}$ (to be compared with adhesive strenghs around $2.5 \mathrm{~N} / \mathrm{m}$ for graphite exfoliation). The technique is similar to the one developed for graphene and weakest adhesive strength wherein a flake is sandwiched between two pieces of tape that are pulled apart. For one exfoliation process, a few tens of small crystals were initially put on a fresh adhesive tape. After around 10 successive exfoliation steps, the flakes were transferred onto Si

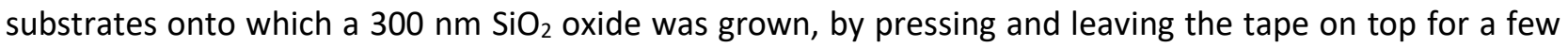
$\mathrm{mn}$ at $\mathrm{T}$ around $70^{\circ} \mathrm{C}$. The thinnest flakes exhibit a thickness around $1 \mathrm{~nm}$, with numerical values close to $0.5 \mathrm{~L}, 1 \mathrm{~L}, 1.5 \mathrm{~L}$ or $2 \mathrm{~L}$, with a typical lateral size of a few $\mu \mathrm{m}$ (see Figure $\mathrm{S} 1 \mathrm{~b}, \mathrm{c}$ for step height determination by AFM). Unfortunately, the exfoliation process results in the presence of large amounts of glue residues on the flake surface. This is not necessarily detrimental to the flake properties, but if one has the purpose of producing ohmic contacts to the flake, then an appropriate process must be used to remove the residues, a problem which in our case is much more stringent than with graphene. With that end in view, up-to-date, UV-resistant synthetic polymers are not the easiest ones to remove, and do not necessarily represent a 
good choice. Among solvents, acetone and xylene are the best, but they only remove part of the glue if used alone, even after the samples are kept several days in acetone at $50^{\circ} \mathrm{C}$. We empirically determined that by using adhesives based on natural rubber, residues turn out to be easier to remove. The MAXene covered Si substrates were then immersed in acetone for $15 \mathrm{~h}$ to dissolve some of the glue. To remove the remainder of the glue the substrates were annealed in high vacuum $\left(10^{-8}\right.$ to $\left.8 \times 10^{-8} \mathrm{mbar}\right)$ at $300^{\circ} \mathrm{C}$ for $3 \mathrm{~h}$. However, even with this process, unwanted residues still cover part of the flake area. The flakes were then stored in a primary vacuum. Crystal growth, size selection and exfoliation as well as process variants are further described in Section S1.

AFM, EFM and KPFM were performed in ambient air with a Brucker Nanoscope $V$ atomic force microscope equipped with EFM and KPFM modules. Most data were obtained using Pt/Ir coated Si tips and a cantilever spring constant $\mathrm{k} \cong 2.8 \mathrm{~N} / \mathrm{m}$, a resonant frequency $\mathrm{f}_{0}$ around $70 \mathrm{kHz}$ and quality factors $\mathrm{Q}$ between 200 and 300. The mechanically-excited oscillation amplitude was set at values ranging from 50 to $100 \mathrm{~nm}$, as estimated by recording approach-retract curves.

The STM measurements were performed under ultrahigh vacuum $\left(\approx 10^{-11} \mathrm{mbar}\right)$ with an OMICRON STM1 instrument operated at room temperature, with home-made $\mathrm{W}$ tips prepared by electrochemical etching. For these measurements, $\mathrm{Cr}_{2} \mathrm{AlC}$ crystals cut into of $5 \mathrm{X} 5 \mathrm{~mm}^{2}$ squares and cleaved in a chamber where a vacuum pressure was $<10^{-9} \mathrm{mbar}$, and then immediately (within a few minutes) transferred under ultrahigh vacuum (below $10^{-11} \mathrm{mbar}$ ) in the STM chamber.

Contact processing of the reported flakes was achieved using conventional e-beam lithography and metal evaporation steps, resulting in $\mathrm{Ti}(4 \pm 1 \mathrm{~nm})+\mathrm{Au}(40 \mathrm{~nm})$ contact lines. For the 4-contact resistance measurements, we used a SR830 DSP lock-in amplifier and current biasing, achieved by adding a $1 \mathrm{M} \Omega$ resistance in series with the devices.

\section{Supporting Information}

Supporting information is available on the Wiley Online Library or from the authors.

\section{Acknowledgements}


A.G., Y.K. and M.W.B. were financially supported by a chair-of-excellence program of the Nanosciences Foundation (Université Grenoble-Alpes Foundation). H. Sellier (Néel Institute) is thanked for pointing an error in the electrostatic computations. Technical support by P. David and V. Guisset for STM measurements, J.F. Motte for e-beam lithography and S. Le Denmat for AFM measurements is acknowledged. Both LMGP and Néel Institute were financially supported by the "Agence Nationale de la Recherche" (project ANR-18-CE09-0041) for the work related to the non-magnetic MAX phases and by the Flag-ERA JTC 2017 project entitled "MORE-MXenes" for the experiments conducted with the $\mathrm{Mo}_{4} \mathrm{Ce}_{4} \mathrm{Al}_{7} \mathrm{C}_{3}$ phase. 


\section{References}

[1] M. Naguib, M. Kurtoglu, V. Presser, J. Lu, J. Niu, M. Heon, L. Hultman, Y. Gogotsi and M. W. Barsoum, Adv. Mater. 2011, 23, 4248.

[2] M.W. Barsoum, MAX phases (Wiley, Weinheim 2013)

[3] M.W. Barsoum, Prog. Solid St. Chem. 2000, 28, 201.

[4] B. Anasori, M.R. Lukatskaya, Y. Gogotsi, Nature Reviews: Materials 2017, 2, 16098.

[5] M. Naguib, O. Mashtalir, J. Carle, V. Presser, J.Lu, L. Hultman, Y. Gogotsi, M.W. Barsoum, ACS Nano 2012, 6, 1322.

[6] J. Halim, K.M. Cook, M. Naguib, P. Eklund, Y. Gogotsi, J. Rosen, M.W. Barsoum, Appl. Surf. Sci. 2016, $362,406$.

[7] J.C. Lei, X. Zhang, Z. Zhou, Front. Phys. 2015, 10, 276.

[8] X. H. Zha, K. Luo, Q.W. Li, Q. Huang, J. He, X.D. Wen and S.Y. Du, EPL 2015, 111, 26007.

[9] M.R. Lukatskaya, O. Mashtalir, C.E. Ren, Y. Dall'Agnese, P. Rozier, P.L. Taberna, M. Naguib, P. Simon, M.W. Barsoum, Y. Gogotsi, Science 2013, 341, 1502.

[10] M. Ghidiu, M. R. Lukatskaya, M. Q. Zhao, Y. Gogotsi, M. W. Barsoum, Nature 2014, 516, 78-U171.

[11] G. Choi, F. Shahzad, Y.M. Bahk, Y.M. Jhon, H. Park, M. Alhabeb, B. Anasori, D.S. Kim, C.M. Koo, Y. Gogotsi, M. Seo, Adv. Opt. Mat. 2018, 6, 1701076.

[12] B. Xu, M. Zhu, W. Zhang, X. Zhen, Z. Pei, Q. Xue, C. Zhi, P. Shi, Adv. Mater. 2016, 28, 3333.

[13] Y. Xie, Y. Dall'Agnese, M. Naguib, Y. Gogotsi, M.W. Barsoum, H.L.L. Zhuang, P.R.C Kent, ACS Nano 2014, $8,9606$.

[14] Y. Xie, M. Naguib, V.N. Mochalin, M.W. Barsoum, Y. Gogotsi, X. Yu, K.W. Nam, X.Q. Yang, A.I. Kolesnikov, P.R.C. Kent, J. Amer. Chem. Soc. 2014, 136, 6385.

[15] M. Naguib, J. Halim, J. Lu, K.M. Cook, L. Hultman, Y. Gogotsi, M.W. Barsoum, J. Amer. Chem. Soc. 2013, $135,15966$.

[16] X. Xie, S. Chen, W. Ding, Y. Nie, Z.D. Wei, Chem Commun. 2013, 49, 10112.

[17] Q.K. Hu, D.D. Sun, Q.H. Wu, H.Y. Wang, L.B. Wang, B.Z. Liu, A.G. Zhou, J.L. He, J. Phys. Chem. A 2013, $117,14253$.

[18] H. Kim, B. Anasori, Y. Gogotsi, H.N. Alshareef, Chem. Mat. 2017, 29, 6472.

[19] H. Lin, S.S. Gao, C. Dai, Y. Chen, J.L. Shi, J. Am. Chem. Soc. 2017, 139, 16235.

[20] T. Ouisse, E. Sarigiannidou, O. Chaix-Pluchery, H. Roussel, B. Doisneau, D. Chaussende, J. Cryst. Growth 2013, 384, 88.

[21] L. Shi, T. Ouisse, E. Sarigiannidou, O. Chaix-Pluchery, H. Roussel, D. Chaussende, B. Hackens, Acta Mater. 2015, 83, 304.

[22] F. Mercier, T. Ouisse, D. Chaussende, Phys. Rev. B 2011, 83, 075411. 
[23] K. S. Novoselov, D. Jiang, F. Schedin, T. J. Booth, V. V. Khotkevich, S. V. Morozov, A. K. Geim, PNAS 2005, $102,10451$.

[24] Q. Tao, T. Ouisse, D. Pinek, O. Chaix-Pluchery, F. Wilhelm, A. Rogalev, C. Opagiste, L. Jouffret, A. Champagne, J.C. Charlier, J. Lu, L. Hultman, M.W. Barsoum and J. Rosen, Phys. Rev. Mat. 2018, 2, 114401.

[25] A. Miranda, J. Halim, A. Lorke, M.W. Barsoum, Mat. Res. Lett. 2017, 5, 322.

[26] T. Ito, D. Pinek, T. Fujita, M. Nakatake, S.I. Ideta, K.Tanaka, T. Ouisse, Phys. Rev. B 2017, 96, 195168.

[27] B.D. Terris, J.E. Stern, D. Rugar, H.J. Mamin, Phys. Rev. Lett. 1989, 63, 2669.

[28] T. Mélin, D. Deresmes, D. Stiévenard, Appl. Phys. Lett. 2002, 81, 5054.

[29] F. Habrard, T. Ouisse, O. Stéphan, M. Armand, M. Stark, S. Huant, E. Dubard, J. Chevrier, J. Appl. Phys. 2004, 96, 7219.

[30] M. Nonnenmacher, M. P. O’Boyle, H. K. Wickramasinghe, Appl. Phys. Lett. 1991, 58, 2921.

[31] H. O. Jacobs, P. Leuchtmann, O. J. Homan, A. Stemmer, J. Appl. Phys. 1998, 84, 1168.

[32] W. Melitz, J. Shen, A.C. Kummel, S. Lee, Surface Sci. Reports 2011, 66, 1.

[33] T. Ouisse, F. Martins, M. Stark, S. Huant, J. Chevrier, Appl. Phys. Lett. 2006, 88, 043102.

[34] T. Ouisse, M. Stark, F. Martins, B. Bercu, S. Huant and J. Chevrier, Phys. Rev. B 2005, 71, 205404.

[35] J. Moser, A. Verdaguer, D. Jiménez, A. Barreiro, A. Bachtold, Appl. Phys. Lett. 2008, 92, 123507.

[36] Young-Jun Yu, Yue Zhao, Sunmin Ryu, L.E. Brus, Kwang S. Kim, P. Kim, Nano Lett. 2009, 9, 3430.

[37] S.S. Datta, D.R. Strachan, E.J. Mele, A.T. C. Johnson, Nano Lett. 2009, 9, 7.

[38] T. Burnett, R. Yakimova, O. Kazakova, Nano Lett. 2011, 11, 2324.

[39] C. Gomez-Navarro, F. J. Guzman-Vazquez, J. Gomez-Herrero, J.J. Saenza, G. M. Sacha, Nanoscale 2012, $4,7231$.

[40] C.K. Oliveira, M.J.S. Matos, M.S.C. Mazzoni, H. Chacham, B.R.A. Neves, Nanotechnology 2012, 23, 175703.

[41] A. Castellanos-Gomez, E. Cappelluti , R. Roldán , N. Agraït, F. Guinea, G. Rubio-Bollinger, Adv. Mater. 2013, 25, 899.

[42] S. Samaddar, J. Coraux, S.C. Martin, B. Grévin, H. Courtois, C.B. Winkelmann, Nanoscale 2016, 8, 15162.

[43] A. Singha, Puspendu Guhab,c, Amrish K. Panwara, Pawan K. Tyagia, Appl. Surf. Sci. 2017, 402, 271.

[44] L.H. Li, T. Tian, Q. Cai, C.J. Shi, E.J.G. Santos, Nat. Comm. 2018, 9, 1271.

[45] Yue Shen, Ying Wang, Yuan Zhou, Chunxi Hai, Jun Hu, Yi Zhang, Beilstein J. Nanotechnol. 2018, 9, 1146.

[46] A. Verdaguer, C. Weis, G. Oncins, G. Ketteler, H. Bluhm, M. Salmeron, Langmuir 2007, 23, 9699.

[47] A. Kurokawa, K. Odaka, Y. Azuma, T. Fujimoto, I. Kojima, Journal of Surface Analysis 2009, 15, 337.

[48] A. Sadeghi, A. Baratoff, S.A. Ghasemi, S. Goedecker, T. Glatzel, S. Kawai, E. Meyer, Phys. Rev. B 2012, $86,075407$.

[49] A. Sadeghi, A. Baratoff, S. Goedecker, Phys. Rev. B 2013, 88, 035436. 
[50] S. Hudlet, M. Saint Jean, C. Guthman, J. Berger, Eur. Phys. J. B 1998, 2, 5.

[51] S. Belaidi, P. Girard, G. Leveque, J. Appl. Phys. 1997, 81, 1023.

[52] T. Ouisse, L. Shi, B. Piot, B. Hackens, V. Mauchamp, D. Chaussende, Phys. Rev. B 2015, 92, 045133.

[53] T. Flatten, F. Matthes, A. Petruhins, R. Salikhov, U. Wiedwald, M. Farle, J. Rosen, D. Bürgler, C.M. Schneider, Appl. Phys. Lett. 2019, 115, 094101.

[54] J. D. Hettinger, S. E. Lofland, T. Meehan, J. Palma, K. Harrel, S. Gupta, A. Ganguly, T. El-Raghy, M. W. Barsoum, Phys. Rev B 2005, 72, 115120.

[55] T. Ouisse, D. Pinek, M.W. Barsoum, Ceramics Int. 2019, 45, 22956.

[56] L. Wang, I. Meric, P. Y. Huang, Q. Gao, Y. Gao, H. Tran, T. Taniguchi, K. Watanabe, L. M. Campos, D. A. Muller, J. Guo, P. Kim, J. Hone, K. L. Shepard, C. R. Dean, Science 2013, 342, 614.

[57] K. Sankaran, K. Moors, S. Dutta, C. Adelmann, Z. Tokei, G. Pourtois, Proc. IEEE International Interconnect Technology Conference (Editor IEEE, 345 E 47 $7^{T H}$ St, New York, NY 10017), 2018, 160.

[58] L. Verger, C. Xu, V. Natu, H.M. Cheng, W. Ren, M. W. Barsoum, Curr. Opin. Solid State Mater. Sci. 2019, 23, 149.
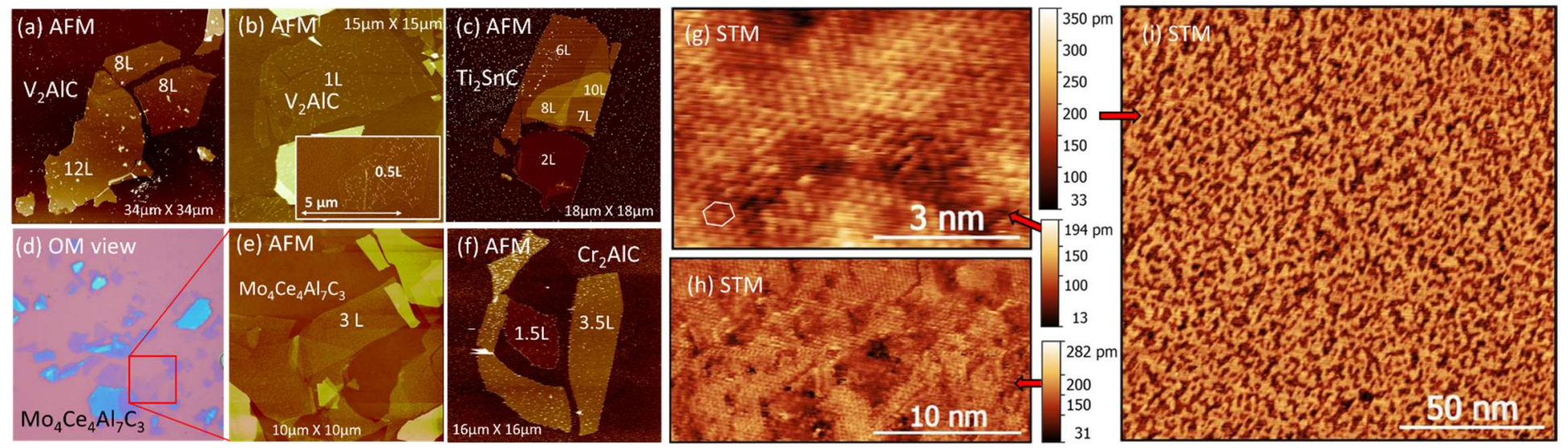

Figure 1. (a) AFM topographic images of MAXene flakes transferred onto $\mathrm{SiO}_{2} / \mathrm{Si}$ substrates. Thickness is presented in multiples of unit cells of each phase. (a) "Large" area, thick $V_{2} A I C$ flakes, (b) unit and sub-unit layer thick $\mathrm{V}_{2} \mathrm{AlC}$ flakes, (c) $\mathrm{Ti}_{2} \mathrm{SnC}$ flakes, (d) OM image of $\mathrm{Mo}_{4} \mathrm{Ce}_{4} \mathrm{Al}_{7} \mathrm{C}_{3}$ flakes, (e) $\mathrm{Mo}_{4} \mathrm{Ce}_{4} \mathrm{Al}_{7} \mathrm{C}_{3}$ flakes and (f) thin $\mathrm{Cr}_{2} \mathrm{AlC}$ flakes. (g), (h) and (i) show STM topographic images of $\mathrm{Cr}_{2} \mathrm{AlC}$ SCs cleaved in UHV and measured in situ at $300 \mathrm{~K}$. (g) Tunneling current $\mathrm{I}=10.2 \mathrm{nA}$ and bias $\mathrm{V}=-0.48 \mathrm{~V}$, (h) I $=3.35 \mathrm{nA}, \mathrm{V}=-0.45 \mathrm{~V}$ and (i) I $=$ $1 \mathrm{nA}, \mathrm{V}=-1 \mathrm{~V}$. 

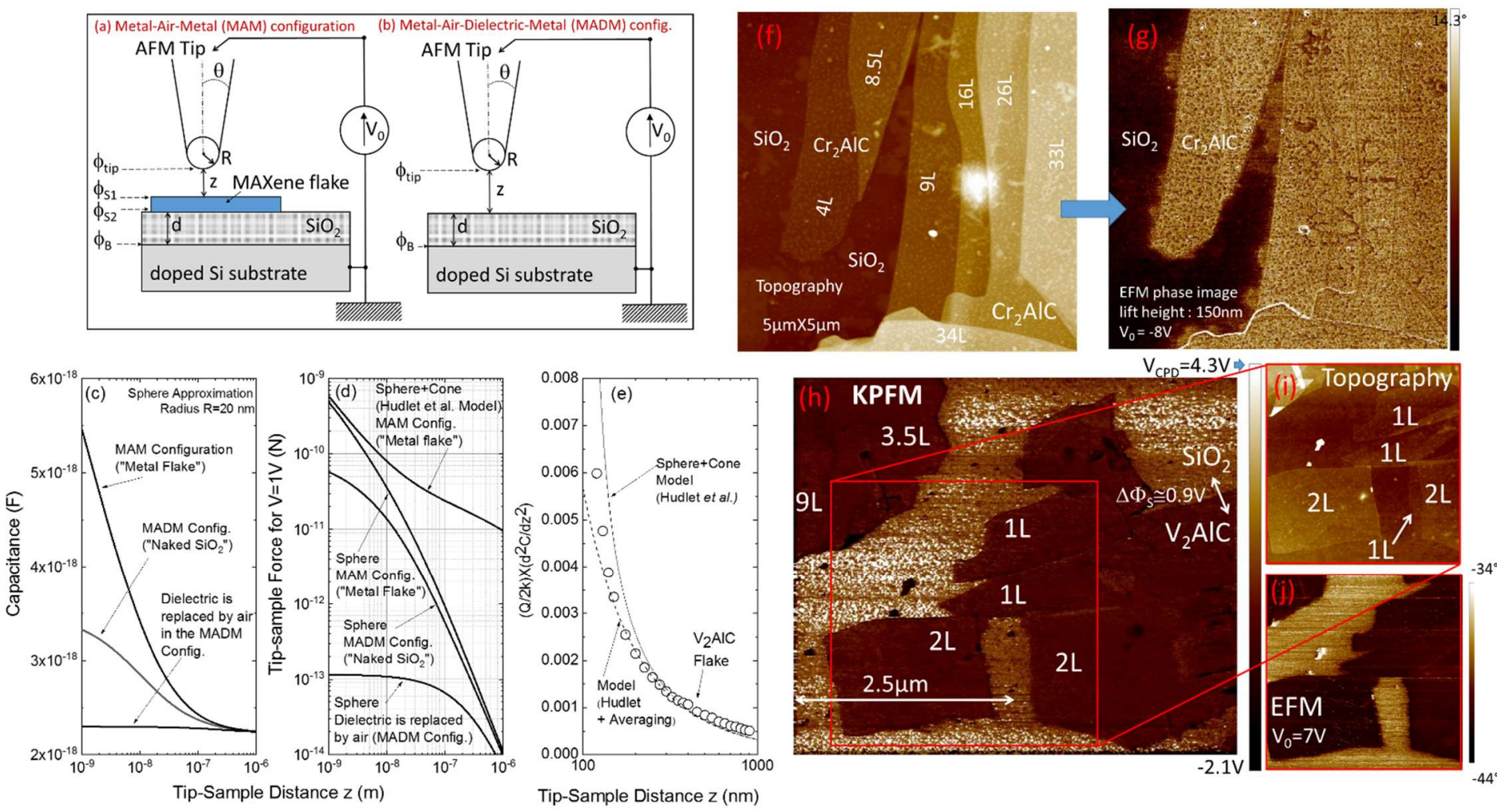

Figure 2. Schematics of the EFM and KPFM configurations with (a) AFM tip positioned above the flake and (b) tip positioned above the $\mathrm{SiO}_{2}$ surface. (c) Theoretical values of the capacitance versus tip-sample distance calculated using the image charge model of Ref.[49] and sphere-plane approximation, showing the difference between the configurations where the tip is positioned above a metallic flake, the $\mathrm{SiO}_{2}$ dielectric or if the dielectric layer is replaced by an air layer with the same thickness. (d) Variation of the electric force with tip-sample distance for a voltage difference of $1 \mathrm{~V}$, calculated in the same cases as in (a), and also using an analytical formula [50] with a sphere and cone approximation, corresponding to the case where the tip is positioned above a metallic flake. (e) Variation of the second derivative of the capacitance versus tipsample distance, where experimental points (circles) are compared to the analytical model [50] (solid line) and to an average of the values computed with the same model over a moving interval equal to the tip oscillation amplitude (dotted line). (f) Topographic image of a $\mathrm{Cr}_{2} \mathrm{AlC}$ MAXene flake exhibiting steps and terraces, covered by a substantial amount of glue residues and (g) EFM phase image measured for a lift height of $150 \mathrm{~nm}$ and a tip voltage of $-8 \mathrm{~V}$. (h) KPFM image of very thin $\mathrm{V}_{2} \mathrm{AlC}$ flakes measured at a lift height of $40 \mathrm{~nm}$, (i) topographic image of a zoomed part of area scanned in (h) and, (j) EFM image of the same area as (i). Variations of both the surface potential and the EFM phase shifts are minor above the flakes, independently of flake thickness. 

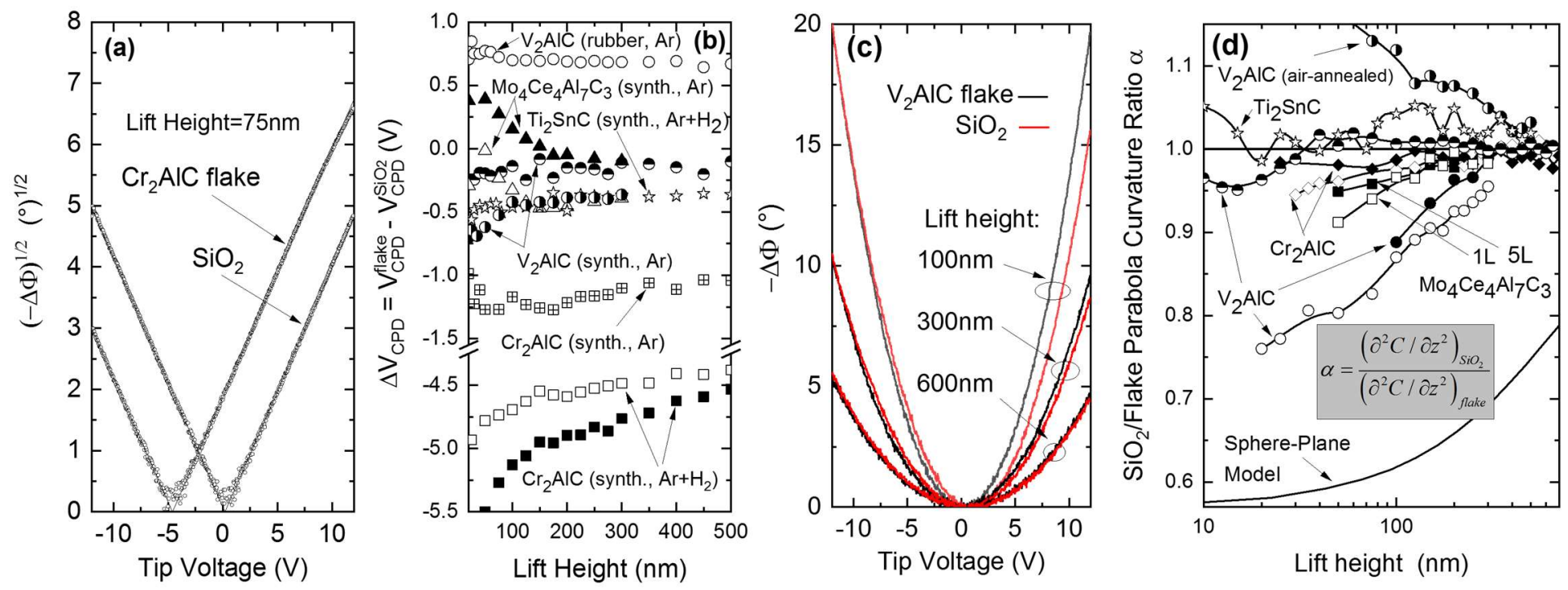

Figure 3. (a) Typical experimental plots of the square root of the phase shift versus tip voltage, measured at a given lift height and a given position, and used to compute a point as that shown in (b). (b) Experimental plot of the contact potential difference between MAXene flakes and the nearby $\mathrm{SiO}_{2}$ surface, measured for different phases, prepared or annealed under various conditions. (c) Typical experimental plots of the parabolas versus tip voltage, with the as a function of lift height, showing marked differences in curvature observed for the flakes or $\mathrm{SiO}_{2}$ as the lift height is reduced to small values. (d) Experimental plot of the capacitance contrast between $\mathrm{MAXene}$ flakes and $\mathrm{SiO}_{2}$ versus lift height, expressed in the form of the ratio $\alpha$ between the electric force parabola's curvatures measured on both surfaces. 

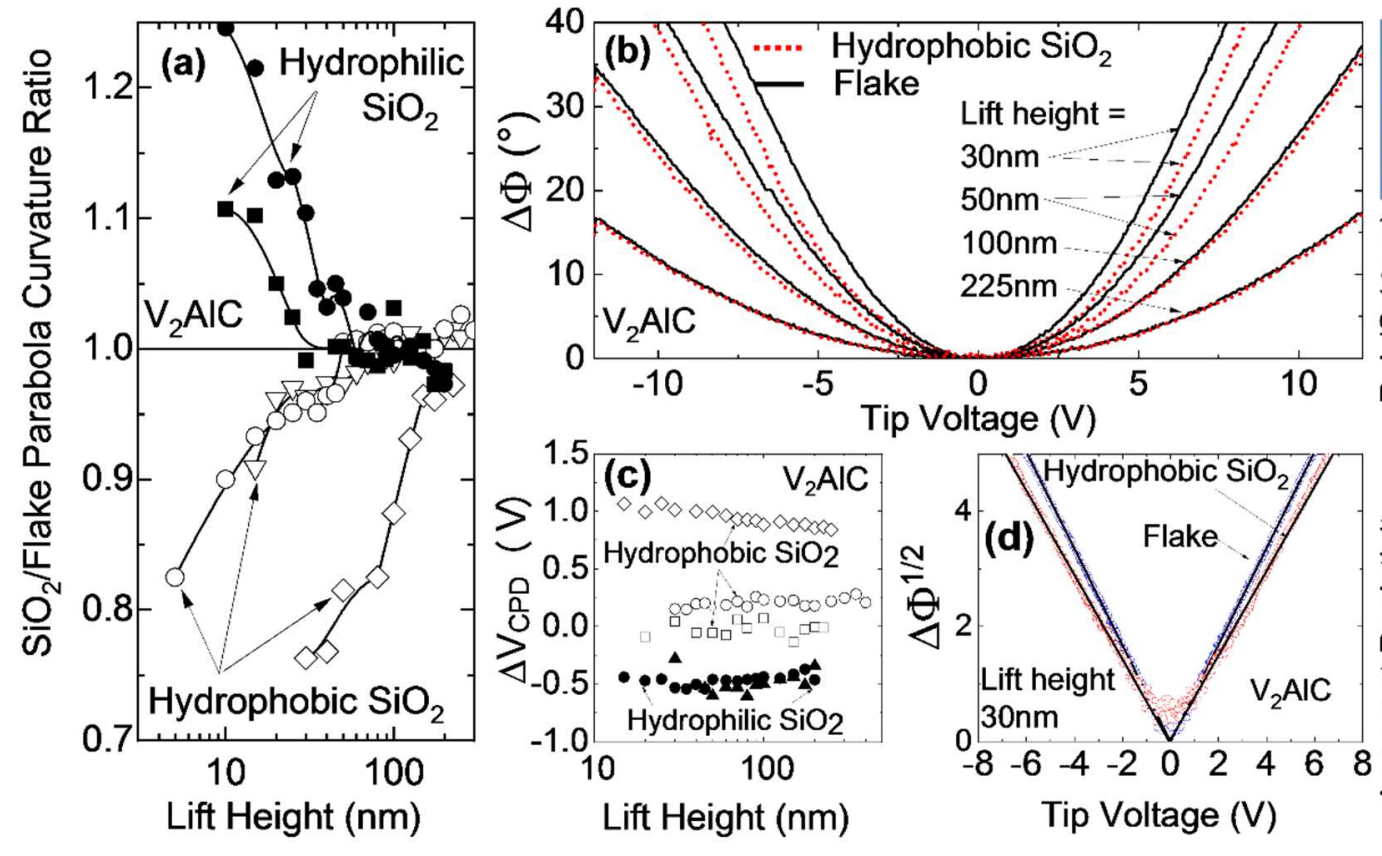

Tip Voltage (V)

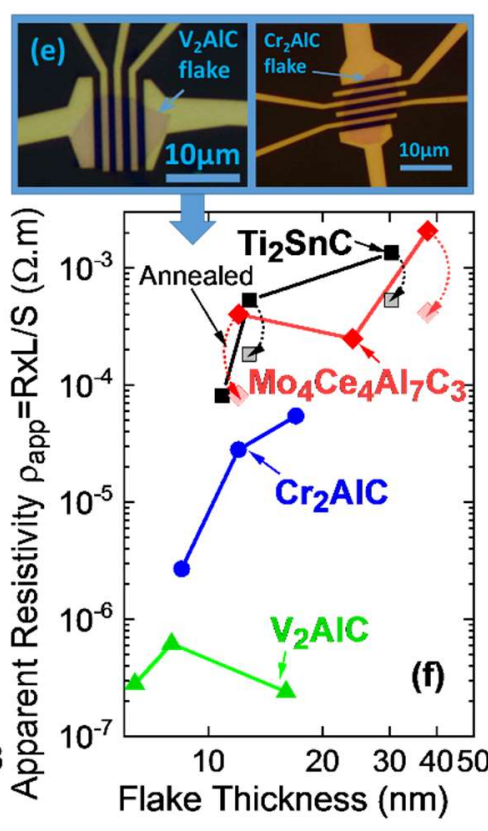

Figure 4. (a) Electric force parabola curvature ratio calculated from phase parabolas EFM capacitance contrast vs. lift height for $\mathrm{V}_{2}$ AIC MAXene flakes prepared under the same conditions, on the same day on hydrophobic or hydrophilic $\mathrm{SiO}_{2}$ surfaces prepared as described in section $\mathrm{S} 3$ and measured on the same day in air with $\mathrm{RH}$ around $45 \%$. (b) Electric force parabolas used to calculate results in a. (c) $V_{C P D} V$ s. lift height. (d) typical square root of the phase shift used to determine the curvature and $V_{C P D}$. (e) OM view of two flakes with electrical contacts. (f) log-log plot of 4-contact apparent resistivity as a function of flake thickness and for various $M A X$ phases and $\mathrm{Mo}_{4} \mathrm{Ce}_{4} \mathrm{AL}_{7} \mathrm{C}_{3}$. Annealing some samples in high vacuum at $300^{\circ} \mathrm{C}$ during $2 \mathrm{~h}$ may lead to a noticeable improvement in resistivity (open symbols).

Table 1. Comparison of typical resistivity values measured at $300 \mathrm{~K}$ from bulk materials and flakes.

\begin{tabular}{|l|c|l|l|}
\hline Material & Resistivity $(\mu \Omega \mathrm{m})$ & \multicolumn{1}{|c|}{ Remarks } & Ref. \\
\hline $\mathrm{Cr}_{2} \mathrm{AlC}$ & $\approx 0.65$ & Polycrystalline samples & {$[2]$} \\
& 0.060 & Single crystal along basal planes & {$[52]$} \\
& 0.27 & Single crystal along basal planes & {$[55]$} \\
& 2.7 & Thin flake $\approx 10 \mathrm{~nm}$ & This work \\
\hline $\mathrm{V}$ AIC & 0.25 & Polycrystalline & {$[54]$} \\
& 0.030 & Single crystal along basal planes & {$[52]$} \\
& 0.28 & Thin flakes $(5$ to $15 \mathrm{~nm})$ & This work \\
\hline
\end{tabular}

